\title{
Assessing the Effects of Parasitism and Predation by Water Mites on the Mosquitoes ${ }^{1}$
}

L. ESTEVA2, Departamento de Matemáticas, FC, UNAM, 04510, México, D.F., México

G. RIVAS, Departamento de Biologia, FC, UNAM, 04510, México, D.F., México

H.M. YANG 4 , Departamento de Matemática Aplicada, IMECC, UNICAMP, Lab-Epifisma, Cx.P. 6065, 13081-970 Campinas, SP, Brasil.

\begin{abstract}
Water mites (Hydrachnidia) are a group of arthropods with a complex life cycle which includes larval and three nymphal stages. The mite larvae parasitize aquatic and semiaquatic insects, while deutonymphs and adult mites are predators of insect larvae and eggs. Since several families of water mites are associated with mosquitoes, there is an interest in the potential use of these mites as biological control agents of Aedes aegypti. The aim of this paper is to use mathematical modelling and analysis to assess the impact of predation and parasitism in the mosquito population by water mites. We discuss the fitness of water mites as biological control of mosquitoes by different scenarios that appear when we change the parasitism and predation parameters. High rates of parasitism and moderate predation can drive two species to a stable coexistence far from the extinction point.
\end{abstract}

\section{Introduction}

Mosquitoes belong to the family Culicidae order Diptera, one of the greatest and diverse groups of Insecta in which there are near 100,000 species described. Some species of the genera Aedes and Anopheles are of great importance in public health since they serve as vectors of several diseases like malaria, dengue and yellow fever. Like most animals, Diptera can be parasitized, and in particular the water mites parasitize the aquatic forms larvae and pupae of insects. Water mites (Hydrachnidia) are an interesting group of microarthropods with nearly 5,000 species described into 50 families, whose habitats are very diverse since they can live in thermal waters, wetlands, temporal pools, and lagoons. The life cycle includes larval stage and three nymphal (nymphocrysalids, deutonymph, and imagochrysalis) stages, which are quiescent except the second one [9].

\footnotetext{
${ }^{1}$ Financial support: FAPESP (Projeto Temático)

${ }^{2}$ lesteva@lya.fciencias.unam.mx

3 grl@hp.fciencias.unam.mx

${ }^{4}$ hyunyang@ime.unicamp.br, fellowship awarded by CNPq.
} 
Water mite larvae need to parasitize aquatic or semiaquatic insects to become nymph, in order to complete the life cycle [9]. On the other hand, deutonymphs and adults depredate insect larvae and, in minor extension, pupae. Thus, there are two significative phases in the population dynamics of the water mites and insects: predation (adult mites eat eggs and larvae of insects) and parasitism (mite larvae parasitize adult insects and, in minor extension, pupae). Larvae of water mites of the family Arrenuridae are the most common ectoparasites of Culicidae sorts of medical importance like Aedes and Anopheles [10].

In general the knowledge about parasite-predation interactions between water mites and mosquitoes is fragmentary. The aim of this study is to evaluate the impact of these relations on the population dynamics of both species. For this goal we formulate a mathematical model in Section 2 for the interactions among the larval and adult stages of mosquitoes and water mites. We discuss the different scenarios that appear when we change the parasitism and predation parameters, and discussion and conclusion are given in Section 3.

\section{The Model}

Laboratory experiments have clearly established that some parasitic water mite (the word water is omitted in this section) species reduce the survival and reproductive rates of their host. It is estimated that mites may reduce the reproductive potential of Masonia perturbans populations by 10\%, and the first gonotrophic cycle of Anopheles crucians parasitized by Arrenurus spp. may be reduced by $35 \%$ [4] [8]. Quantitative studies on the feeding behavior of mites are scarce. The only records of mites feeding on mosquitoes are from visual observations in laboratory. Although there are some species like Limnesia jamurensis that are great predators of mosquito larvae (each mite consumed roughly 6 to 8 larvae per day) [3], in general mites are not specific predaceous of mosquitoes. For instance, Arrenurus species also feed on ostracods [8]. We consider the interaction between mosquito and mite populations. The mosquito population is divided in two stages: the immature (larvae and pupae) and the adult form. We denote by $P$ the population size of the immature phases of the insect at time $t$. The adult mosquitoes are divided in healthy $M$ and parasitized $W$. On the other hand, we will consider only the life stages of mites where they parasitize or depredate the mosquito population. One is mite larvae $L$ that parasitize adult mosquitoes, and other is predator mites $A$ consisting of deutonymphs (pupae) and adult mites that predate mosquito larvae.

With respect to vital dynamics, the per capita mortality rates of immature mosquitoes $P$, healthy mosquitoes $M$ and parasitized mosquitoes $W$ are assigned by $\mu_{P}, \mu_{M}$ and $\mu_{W}$, respectively. Since parasitism could increase mortality of mosquitoes, we assume $\mu_{M} \leq \mu_{W}$. On the other hand, the per capita mortality rates of mite larvae $L$ and depredator mites $A$ are given by $\mu_{L}$ and $\mu_{A}$, respectively. For both populations, the net oviposition rate per female insect is proportional to their density, but it is also regulated by a carrying capacity depending on the occupation of the available breeder sites. In this model we assume that the per capita oviposition rates of healthy and parasitized mosquitoes are given by $\phi_{M}\left(1-P / C_{M}\right)$ 
and $\phi_{W}\left(1-P / C_{M}\right)$, respectively, where $C_{M}$ is the carrying capacity related to the amount of available nutrients and space, and $\phi_{M}$ and $\phi_{W}$ are the corresponding intrinsic oviposition rates. According to the biological observations, parasitism reduces the birth capacity of mosquitoes, thus we assume that $\phi_{W} \leq \phi_{M}$. Analogously, the per capita oviposition rate of mites is $\phi_{A}\left(1-L / C_{A}\right)$, where $\phi_{A}$ is the intrinsic oviposition rate and $C_{A}$ is the carrying capacity for mites. Finally, the immature mosquito population becomes adult insects at a per capita rate $\alpha$.

Hypotheses about the interaction between mite and mosquito populations are the following. Mosquito larvae are assumed to decrement due to predation by deutonymphs and adult mites proportionally to the product of the size of both populations, $k_{2} P A$, where $k_{2}$ is the per capita predation rate. Predation is responsible for the development of deutonymphs to adult mites, which in turn lay eggs. This effect is introduced in the life expectancy of adult mites, assuming that the fitness of adult mites is increased by a term $q k_{2} P A$, with $0 \leq q \leq 1$. In other words, the decreasing of mosquito larvae by $k_{2} P A$ increments the fitness of mites by $\mu_{A}-q k_{2} P A$. Notice that the parameter $q$ must be correlated to the predation rate $k_{2}$ in order to avoid overall negative mortality rate for adult mites. We recall that mite larvae need to parasite mosquitoes in order to complete their life cycle. We assume that mite larvae search independently and randomly, with a constant efficiency. Then, following [6] the rate of parasitism of mosquitoes by mite larvae is proportional to $M\left(1-e^{-a L}\right)$, but, in order to simplify the model, we will approximate by $a M L$, where $a$ is the searching efficiency of mite larvae. Assuming that $k_{1} M$ is the per capita rate of parasitism by larvae to develop to next stage, the flow from the larval stage $L$ to the adult stage $A$ is given by $k_{1} M L$, where $k_{1}=a b$, with $b$ being a constant with dimension $[\text { time }]^{-1}$. Hence, adult mosquitoes $M$ become parasitized mosquitoes $W$ at the same rate $k_{1} M L$.

According to the assumptions above, the model is given by

$$
\left\{\begin{array}{l}
P^{\prime}=\phi_{M}\left(1-\frac{P}{C_{M}}\right) M+\phi_{W}\left(1-\frac{P}{C_{M}}\right) W-\left(\alpha+\mu_{P}\right) P-k_{2} P A \\
M^{\prime}=\alpha P-k_{1} M L-\mu_{M} M \\
W^{\prime}=k_{1} M L-\mu_{W} W \\
L^{\prime}=\phi_{A}\left(1-\frac{L}{C_{A}}\right) A-\mu_{L} L-k_{1} M L \\
A^{\prime}=k_{1} M L-\left(\mu_{A}-q k_{2} P\right) A,
\end{array}\right.
$$

where all parameters of the model are non-negative. First and last equations are strongly coupled, showing that there is a trade-off between predation and fitness, which can lead to oscillating behavior for some range of parameters. It can be shown that solutions starting in the region

$$
\Omega=\left\{(P, M, W, L, A) \in R_{+}^{5}: 0 \leq P \leq C_{M}, 0 \leq L \leq C_{A}\right\}
$$

remain there for all $t \geq 0$. Therefore, $\Omega$ is positively invariant under above system, and it is sufficient to consider solutions in this region where the usual existence, uniqueness and continuation results hold for the system.

The equilibria of system (2.1), which belong to the boundary of $\Omega$, are: $P_{0}=$ 
$(0,0,0,0,0)$ and $P_{M}=\left(P^{*}, M^{*}, 0,0,0\right)$, where

$$
\left\{\begin{array}{l}
P^{*}=\left(1-\frac{1}{R_{M}}\right) C_{M} \\
M^{*}=\frac{\alpha}{\mu_{M}} P^{*}
\end{array}\right.
$$

and

$$
R_{M} \equiv \frac{\alpha}{\alpha+\mu_{P}} \times \frac{\phi_{M}}{\mu_{M}} .
$$

The equilibrium $P_{0}$ is the situation where both species are absent and $P_{M}$, the state where only mosquitoes are present. From the expression for $P^{*}$, it follows that $P_{M}$ will be feasible if and only if $R_{M}>1$. The parameter $R_{M}$ is the basic offspring number of mosquitoes, that is, the average number of mosquitoes produced by a single mosquito. This can be seen as follows: since $1 /\left(\alpha+\mu_{P}\right)$ is the average time of survival of an immature mosquito and $1 / \alpha$ is the average time of its permanence as such, then $\alpha /\left(\alpha+\mu_{P}\right)$ is the probability that an egg will succeed to become an adult mosquito. On the other hand, $\phi_{M} / \mu_{M}$ is the average number of eggs oviposited by one mosquito. Thus, the product of the last two quantities, which is equal to $R_{M}$, is the average number of mosquitoes produced by a single mosquito. Then, $P_{M}$ will be feasible if and only if the basic offspring number is bigger than one.

The stability properties of $P_{0}$ are given by the eigenvalues of the derivative of System (2.1) evaluated at this point, which is given by

$$
D F\left(P_{0}\right)=\left[\begin{array}{ccccc}
-\left(\alpha+\mu_{P}\right) & \phi_{M} & \phi_{W} & 0 & 0 \\
\alpha & -\mu_{M} & 0 & 0 & 0 \\
0 & 0 & -\mu_{W} & 0 & 0 \\
0 & 0 & 0 & -\mu_{L} & \phi_{A} \\
0 & 0 & 0 & 0 & -\mu_{A}
\end{array}\right] .
$$

Solving $\operatorname{Det}\left(\lambda I-D F\left(P_{0}\right)\right)=0$, we find that the eigenvalues are $-\mu_{A},-\mu_{L},-\mu_{W}$ and the roots of the polynomial

$$
p_{1}(\lambda)=\lambda^{2}+\left(\alpha+\mu_{P}+\mu_{M}\right) \lambda+\left(\alpha+\mu_{P}\right) \mu_{M}\left(1-R_{M}\right) .
$$

The roots of $p_{1}$ have negative real part if and only if its coefficients are positive, and it is clear that this is equivalent to the condition $R_{M}<1$. Then, the trivial equilibrium $P_{0}$ is locally asymptotically stable if $R_{M}<1$ and a saddle point if $R_{M}>1$.

Global stability of $P_{0}$ in $\Omega$ is proven when $R_{M} \leq 1$. Let us define the Lyapunov function $V_{1}: \Omega \rightarrow R$ given by

$$
V_{1}=\alpha_{P} P+\left(\alpha+\mu_{P}\right)(M+W) .
$$

The orbital derivative of $V_{1}$ is given by

$$
\dot{V}_{1}=-\left(\alpha+\mu_{P}\right)\left[\mu_{M}\left(1-R_{M}(1-P / C)\right)-\mu_{W}\left(1-r R_{M}(1-P / C)\right)\right],
$$

where $r=\frac{\phi_{W} \mu_{M}}{\phi_{M} \mu_{W}} \leq 1$ by hypothesis. If $R_{M} \leq 1$ then $\dot{V}_{1} \leq 0$ and, from inspection of system (2.1), it can be seen that the maximal invariant set contained in $\dot{V}_{1}=0$ is $P_{0}$ 
which is locally asymptotically stable. Therefore, it follows from La-Salle Lyapunov Theorem [2] that $P_{0}$ is globally asymptotically stable in $\Omega$.

The equilibrium $P_{M}$ is feasible when $R_{M}>1$, and its stability is determined by the eigenvalues of the matrix $D F\left(P_{M}\right)$ given by

$$
\left[\begin{array}{ccccc}
-\frac{\phi_{M}}{C_{M}} M^{*}-\left(\alpha+\mu_{P}\right) & \phi_{M}\left(1-\frac{P^{*}}{C_{M}}\right) & \phi_{W}\left(1-\frac{P^{*}}{C_{M}}\right) & 0 & k_{2} P^{*} \\
\alpha & -\mu_{M} & 0 & -k_{1} M^{*} & 0 \\
0 & 0 & -\mu_{W} & k_{1} M^{*} & 0 \\
0 & 0 & 0 & -\mu_{L}-k_{1} M^{*} & \phi_{A} \\
0 & 0 & 0 & k_{1} M^{*} & -\mu_{A}+q k_{2} P^{*}
\end{array}\right] \text {. }
$$

After some calculations, we find that the eigenvalues of $D F\left(P_{M}\right)$ are $-\mu_{W}$ and the roots of the polynomials

$$
\left\{\begin{array}{l}
p_{2}(\lambda)=\lambda^{2}+\left(\frac{\phi_{M}}{C_{M}} M^{*}+\alpha+\mu_{P}+\mu_{M}\right) \lambda+\mu_{M}\left(\alpha+\mu_{P}\right)\left(R_{M}-1\right) \\
p_{3}(\lambda)=\lambda^{2}+\left(k_{1} M^{*}+\mu_{L}+\mu_{A}-q k_{2} P^{*}\right) \lambda+\left(k_{1} M^{*}+\mu_{L}\right)\left(\mu_{A}-q k_{2} P^{*}\right)-\phi_{A} k_{1} M^{*} .
\end{array}\right.
$$

The coefficients of the polynomial $p_{2}$ are positive (we have $R_{M}>1$ ) which in turn implies that its roots have negative real part. On the other hand, the polynomial $p_{3}$ has roots with negative real part if and only if

$$
\left\{\begin{array}{l}
k_{1} M^{*}+\mu_{L}+\mu_{A}-q k_{2} P^{*}>0 \\
\left(k_{1} M^{*}+\mu_{L}\right)\left(\mu_{A}-q k_{2} P^{*}\right)>\phi_{A} k_{1} M^{*} .
\end{array}\right.
$$

The above conditions are satisfied if and only if

$$
\mu_{A}>\mu_{A}^{t h} \equiv q k_{2} P^{*}=q k_{2}\left(1-\frac{1}{R_{M}}\right) C_{M},
$$

where $\mu_{A}^{t h}$ is the threshold predating mite population mortality rate (or fitness), and

$$
R_{A} \equiv \frac{\phi_{A}}{\mu_{A}-q k_{2} P^{*}} \times \frac{k_{1} M^{*}}{k_{1} M^{*}+\mu_{L}}<1,
$$

where $R_{A}$ is the basic offspring number of mites. Note that if $\mu_{A}<\mu_{A}^{\text {th }}$, we have $R_{A}<0<1$. Hence the stability of $P_{M}$ is summarized by $R_{A}<1$.

Notice that $q k_{2} P^{*}$ represents the equilibrium per capita rate at which the adult mite population predates the mosquito larvae at the equilibrium $P^{*}$. On the other hand, the left hand side of inequality (2.9) can be interpreted as the average number of eggs laid by a single predating mite that survive to the adult stage (first term) after being succeeded to parasitize mosquitoes (second term) when the number of larvae and adult mosquitoes are at the equilibriums $P^{*}$ and $M^{*}$, respectively. Then, assuming that mosquito population is at the equilibrium $P_{M}$, conditions (2.8) and (2.9) say that if the fitness due to predation by the mite population does not surpass the natural mortality rate $\left(\mu_{A}^{t h}<\mu_{A}\right)$ and the average number of adult mites resulting from a single mite is less than one $\left(R_{A}<1\right)$, only the mosquito population can survive. In terms of $k_{1}$ and $k_{2}$, the summarizing condition $\left(R_{A}<1\right)$ of stability of $P_{M}$ is

$$
k_{2}<\frac{1}{q P^{*}}\left(\mu_{A}-\frac{\phi_{A} k_{1} M^{*}}{k_{1} M^{*}+\mu_{L}}\right) .
$$


This implies that $P_{M}$ is stable in the region of the parameters space $k_{1} k_{2}$ below the hyperbola given by the right hand side of (2.10) which has asymptote $k_{2}=$ $\left(\mu_{A}-\phi_{A}\right) /\left(q P^{*}\right)$. Note that this asymptote is negative for $\phi_{A}>\mu_{A}$ (we have $k_{1}^{t h}$ above which $P_{M}$ is always unstable disregarding the predation parameter $k_{2}$ ) and positive for $\phi_{A}<\mu_{A}$ (we do not have $k_{1}^{t h}$, which implies that $P_{M}$ is stable in the region between the axis- $k_{1}$ and hyperbola).

Global stability of $P_{M}$ can be proved in the special case $q=0$ and $\phi_{A} \leq \mu_{A}$ via Lyapunov function defined in $\Omega$ as $V_{2}=L+A$, with orbital derivative

$$
\dot{V}_{2}=\left[\phi_{A}\left(1-L / C_{A}\right)-\mu_{A}\right] A-\mu_{L} L \leq 0 .
$$

All solutions starting in $\Omega$ approach the maximal invariant set contained in $\dot{V}_{2}=0$ [2]. It can be seen readily that this set is the the projection of $\Omega$ on the plane $W=A=L=0$. Hence equations of system (2.1) are reduced in the following two dimensional system in $P$ and $M$, given by

$$
\left\{\begin{array}{l}
P^{\prime}=\phi_{M}\left(1-\frac{P}{C_{M}}\right) M-\left(\alpha+\mu_{P}\right) P \\
M^{\prime}=\alpha P-\mu_{M} M .
\end{array}\right.
$$

Applying Bendixon criteria [11] it can be seen that this system does not have periodic orbits, and since $P_{M}$ is locally asymptotically stable, all trajectories with initial conditions $P(0)$ and $M(0)$ different from zero approach this equilibrium. Therefore $P_{M}$ is globally asymptotically stable in $\Omega$ minus the plane $P=M=0$. This special case describes water mites predating their host, but there is not any advantage in the fitness of mites.

When mosquito population is zero $\left(P_{0}\right)$ then mites can not complete their life cycle implying that they will not be able to survive, because mite larvae is specific parasite of mosquitoes. This is always true when $R_{M}<1$, that is, neither mosquitoes are able to invade and colonize a specified habitat. The equilibrium $P_{M}$ portraits the mite population being not able to survive in the habitat where mosquitoes are well established. In this case, even for $R_{M}>1$, mite population failed to be good predator $\left(\mu_{A}<\mu_{A}^{t h}\right)$ or despite of being a good predator $\left(\mu_{A}>\mu_{A}^{t h}\right)$, mite population failed to produce sufficient offsprings due to predation and parasitism $\left(R_{A}<1\right)$. Next we analyze the effect of remaining condition $R_{M}>1$ and $R_{A}>1$ (predation helps to increase the survival, or fitness, of mites above the threshold, $\left.\mu_{A}>\mu_{A}^{t h}\right)$ on the dynamic system.

The coordinates $\bar{M}, \bar{W}$ and $\bar{A}$, in terms of $\bar{L}$ and $\bar{P}$, of the non trivial equilibrium point $P_{M A}$ of system (2.1) are given by

$$
\left\{\begin{array}{l}
\bar{M}=\frac{\alpha}{k_{1} \bar{L}+\mu_{M}} \bar{P} \\
\bar{W}=\frac{k_{1}}{\mu_{W}} \times \frac{\alpha}{\left(k_{1} \bar{L}+\mu_{M}\right)} \bar{P} \bar{L} \\
\bar{A}=\frac{k_{1}}{\left(\mu_{A}-q k_{2} \bar{P}\right)} \times \frac{\alpha}{\left(k_{1} \bar{L}+\mu_{M}\right)} \bar{P} \bar{L} .
\end{array}\right.
$$

This equilibrium has biological meaning for $0<\bar{L}<C_{A}$ and $0<\bar{P}<\mu_{A} /\left(q k_{2}\right)$. Substituting (2.11) in the first and third equations of (2.1) and solving for $\bar{L}$, we 
obtain after some manipulations that the solutions $(\bar{P}, \bar{L})$ are the intersections of the graphs of the functions

$$
\left\{\begin{aligned}
f(\bar{P}) & =\frac{\mu_{M}\left(R_{M}-1-R_{M} \bar{P} / C_{M}\right)\left(\mu_{A}-q k_{2} \bar{P}\right)}{k_{1}\left[\left(1-r R_{M}+r R_{M} \bar{P} / C_{M}\right)\left(\mu_{A}-q k_{2} \bar{P}\right)+\alpha k_{2} \bar{P} /\left(\alpha+\mu_{P}\right)\right]} \\
g(\bar{P}) & =\frac{\alpha \phi_{A} k_{1} \bar{P}-\left(\mu_{L} \mu_{M}+k_{1} \alpha \bar{P}\right)\left(\mu_{A}-q k_{2} \bar{P}\right)}{k_{1}\left(\mu_{A}-q k_{2} \bar{P}+\alpha \phi_{A} \bar{P} / C_{A}\right)} .
\end{aligned}\right.
$$

We observe that if $R_{M} \leq 1$ then $f(\bar{P}) \leq 0$ for $\bar{P} \in\left[0, \mu_{A} /\left(q k_{2}\right)\right]$, therefore $R_{M}>1$ is a necessary condition for positive solutions of (2.12), and in the following we assume it. Let $f_{1}(\bar{P})$ denote the denominator in the expression for $f(\bar{P})$. Since $f_{1}\left(\mu_{A} /\left(q k_{2}\right)\right)>0$, and $\lim _{P \rightarrow \infty} f_{1}(\bar{P})=-\infty$, one of the zeros of $f_{1}$ is at the right of $\mu_{A} /\left(q k_{2}\right)$. The other zero, denoted by $P_{f}$, is negative if $f_{1}(0)>0$ (in particular this holds if $\left.r R_{M} \leq 1\right)$, and it is in the interval $\left[0, \mu_{A} /\left(q k_{2}\right)\right]$ if $f_{1}(0) \leq 0$. Now, define $P_{\min }=\max \left\{0, P_{f}\right\}$ and $P_{\max }=\min \left\{P^{*}, \mu_{A} /\left(q k_{2}\right)\right\}$, where $P^{*}$ is given by (2.2). It is a simple matter to prove that $f(\bar{P})$ is a positive decreasing function for $P_{\min }<\bar{P}<P_{\max }$, and it is negative if $P_{\min }>0$ and $0<\bar{P}<P_{\min }$ or $P_{\max }<\mu_{A} /\left(q k_{2}\right)$ and $P_{\max }<\bar{P}<\mu_{A} /\left(q k_{2}\right)$. This implies that the coordinate $\bar{P}$ of the solution of $(2.12)$, if it exists, should be in the interval $\left(P_{\min }, P_{\max }\right)$. Furthermore,

$$
\begin{cases}f\left(P_{\text {min }}\right)=\frac{\mu_{A} \mu_{M}\left(R_{M}-1\right)}{k_{1}\left[\left(1-r R_{M}\right) \mu_{A}+\alpha k_{2} /\left(\alpha+\mu_{P}\right)\right]}>0, & \text { if } P_{\text {min }}=0, \\ \lim _{P \rightarrow P_{\text {min }}^{+}} f(\bar{P})=\infty, & \text { if } P_{\text {min }}=P_{f}, \\ \lim _{P \rightarrow P_{\text {min }}} f(\bar{P})=-\infty, & \text { if } P_{\text {min }}=P_{f}, \\ f\left(P_{\text {max }}\right)=0 . & \end{cases}
$$

Next, we analyze the function $g(P)$. It is easy to verify that $g(P)$ has one zero $P_{g}$ in the interval $\left[0, \mu_{A} /\left(q k_{2}\right)\right]$, is increasing for $P_{g} \leq \bar{P} \leq \mu_{A} /\left(q k_{2}\right)$ and $g\left(\mu_{A} /\left(q k_{2}\right)\right)=C_{A}$. Therefore, if $P_{\max }=\mu_{A} /\left(q k_{2}\right)$, equations $(2.12)$ have a unique solution $(\bar{P}, \bar{L})$ with $\bar{P} \in\left(\max \left\{P_{f}, P_{g}\right\}, \mu_{a} / q k_{2}\right)$. Now, assume $P_{\max }=P^{*}$. Substituting $\alpha P^{*}$ by $\mu_{A} M^{*}$ in $g\left(P^{*}\right)$, we obtain

$$
g\left(P^{*}\right)=\frac{\mu_{M}\left[\phi_{A} k_{1} M^{*}-\left(k_{1} M^{*}+\mu_{L}\right)\left(\mu_{A}-q k_{2} P^{*}\right)\right]}{k_{1}\left(\mu_{A}-q k_{2} P^{*}+\mu_{A} \phi_{A} M^{*}\right)} .
$$

This value is bigger than zero if and only if

$$
R_{A} \equiv \frac{\phi_{A}}{\mu_{A}-q k_{2} P^{*}} \times \frac{k_{1} M^{*}}{k_{1} M^{*}+\mu_{L}}>1,
$$

Then, in the case $\mu_{A}>\mu_{A}^{t h}=q k_{2} P^{*}$, there exists a unique positive solution of (2.12) with $\bar{P} \in\left(\max \left\{P_{f}, P_{g}\right\}, \bar{P}\right)$ only when condition (2.13) holds.

In the parameters space $k_{1} k_{2}$ the region of existence of $P_{M A}$ correspond the points $\left(k_{1}, k_{2}\right)$ above the hyperbola given by equation (2.10). Writing inequality (2.13) in the equivalent form

$$
k_{1}>\frac{\mu_{L}\left(\mu_{A}-q k_{2} \bar{P}\right)}{M^{*}\left(\phi_{A}-\left(\mu_{A}-q k_{2} \bar{P}\right)\right)}=k_{1}^{*},
$$


we obtain a lower bound $k_{1}^{*}$ of the rate of parasitism necessary for coexistence of both species in terms of their demographic parameters and the rate $k_{2}$ of predation. The parameter $R_{W}=r R_{M}$ represents the number of adult mosquitoes produced by a parasitized mosquito. When $r$ decreases, it is expected that the number of larvae $\bar{P}$ (and consequently adult mosquitoes $\bar{M}+\bar{W}$ ) decreases.

The equilibrium $P_{M A}$ could not be found explicitly and its local stability is very difficult to determine since we must show that the eigenvalues of the $5 \times 5$ jacobian matrix at $P_{M A}$ have negative real part. Nevertheless, numerical simulations suggest that this equilibrium is stable under suitable conditions.

\section{Discussion and Conclusion}

Most of the studies in population dynamics of mites have been done on species that are important for humans, as it is the case of ticks of cattle, mites predators of other plague mites of different cultures, and ectoparasitic mites of bees like of Varroasis. Nevertheless, for water mites and their interaction with insects, these studies are scarce, being limited mainly to the determination of some demographic parameters. For example, there are evidences that parasitism of water mites on Culicidae affect the metabolism of females of this species with consequent reduction in their birth rate [5]; however, there are no quantitative results and only a few field and experimental observations show that the damage on the mosquito population is not so strong to drive it to extinction. On the other hand, there is a predator-prey relationship between water mites and Culicidae since this species larvae are part of the potential food of deutonymphs and adult mites, thus population dynamics of the water mites has a substantive dependency with respect to the mosquitoes. But still the measurements of how the predation by water mites affect the dynamics of mosquito populations are insufficient.

In this paper we propose and analyze a mathematical model for the parasitism and predation between water mites and mosquitoes. These ecological interactions are modeled by the mass action law. The model incorporates the larval and adult stages of the two populations. We also include a class of parasitized mosquitoes which have a lower birth rate and higher mortality rate than healthy mosquitoes. We assumed that the predation increases the survival rate of mites. However, elsewhere [1] the increasing in the reproductive rate was assumed.

Under the biological assumptions, our model has three equilibrium points. The trivial equilibrium point $P_{0}$, to which the system evolves if the basic offspring number of mosquito population is such that $R_{M} \leq 1$. When $R_{M}>1$ a second equilibrium $P_{M}$ emerges, where only mosquito population is present when $R_{A}<1$. The absence of the mosquitoes is a limiting factor for water mites, since without mosquitoes they can not complete their life cycle. For this reason there is not an equilibrium where only water mites are present.

Stability analysis of $P_{M}$ reveals that this equilibrium is stable when the increasing of water mite population due to predation is less than their mortality rate, and the average number of water mites resulting from a single water mite is less than one, according to inequalities (2.8) and (2.9). When the conditions of stability of 
$P_{M}$ not longer hold, we found an interior equilibrium where two species coexist $\left(R_{M}>1\right.$ and $\left.R_{A}>1\right)$. The behavior of the populations can be interpreted in terms of the bifurcation diagram with respect to $k_{1} k_{2}$, according to equation (2.10). If the oviposition rate of water mites $\phi_{A}$ is less than their mortality rate $\mu_{A}$, and predation rate $k_{2}$ is not sufficiently high (below the asymptote), the water mite population would not be able to survive, no matter the value of the parasitism rate $k_{1}$ and the initial state of the population. Moreover, high rates of predation will drive the system to coexistence of the two populations even for low rates of parasitisms. In the case $\phi_{A}>\mu_{A}$, coexistence can be reached if parasitism is sufficiently high $\left(k_{1}>\frac{\mu_{A} \mu_{L} M^{*}}{\phi_{A}-\mu_{A}}\right)$, even the predation is absent. In either case we could conclude that the dynamics of mosquito and water mites populations are more sensitive to predation than to parasitism in the sense that for any value of $k_{1}$, it is enough to increment $k_{2}$ sufficiently to enter to the coexistence region.

It is clear that if the oviposition rate of water mites is less than the mortality rate, this population can not survive even if they are able to complete the life cycle via parasitism. Predation, however, gives the opportunity to the water mite population to increase. On the other side, if the oviposition rate is bigger than the mortality, the basic offspring of water mites is bigger than one and predation is not essential to their surviving. This result is according to the fact that mosquitoes are not the only food source of water mites (other sources can be microcrustaceans or other species of mites [9]). Some biological studies suggest that water mites species could be a potential biological control of mosquito populations. In [8] it is mentioned that Arrenurus mite parasitism appears to have a significant detrimental effect on certain Aedes species of mosquitoes that are common in Canada. Acarine genera predators of mosquitoes that could be biological controls are Limnesia that consume large amounts of mosquito eggs and Piona that is a voracious predator of mosquito larva [7].

Finally, we think that mathematical models jointly with experimental data could be a powerful tool to address important questions concerning the dynamical interactions of water mites and mosquitoes, and to assess the effectivity of biological control of mosquitoes using water mites.

Resumo. Ácaros aquáticos (Hydrachnidia) são um grupo de artrópodos com um ciclo de vida complexo que inclui fase larval e três estágios de ninfa. As larvas de ácaros parasitam insetos aquáticos e semi-aquáticos, enquanto deutoninfas e ácaros adultos são predadores de larvas e ovos. Como muitas famílias de ácaros aquáticos estão associadas a mosquitos, existe um grande interesse em estudar o potencial uso desses ácaros como controladores biológicos de Aedes aegypti. O objetivo do trabalho é analisar e determinar, por meio de modelagem matemática, o impacto de parasitismo e predatismo na população de mosquitos pelos ácaros. Discute-se diferentes cenários que surgem quando se variam parâmetros relacionados com predatismo e parasitismo. Taxa elevada de parasitismo e taxa moderada de predatismo podem resultar na co-existância das duas espécies longe da extinção de ambas. 
Esteva, Rivas and Yang

\section{References}

[1] L. Esteva, G. Rivas, H.M. Yang, Modelling parasitism and predation of mosquitoes by water mites, J. Math. Biol., 53 (2006), 540-555.

[2] J.K. Hale, "Ordinary Differential Equations", John Wiley and Sons, New York, 1969.

[3] M. Laird, Some natural enemies of mosquitoes in the vicinity of Palmalmal, New Britain, Trans. R. Soc. N.Z., 76 (1947), 453-476.

[4] C.A. Lanciani, A.D. Boyt, The effect of a parasitic water mite, Arrenurus pseudotenuicollis (Acari: Hydrachnellae), on the survival and reproduction of the mosquito Anopheles crucians (Diptera: Culicidae), J. Med. Ent., 14 (1977), 10-15.

[5] G. Mullen, Acarine parasites of mosquitoes I. A critical review of all know records of mosquitoes parasitized by mites (Acarina: Hydrachnellae), Proc. N.J. Mosq. Exterm. Assoc., 61 (1975), 90-94.

[6] A.J. Nicholson, V.A. Bailey, An outline of the dynamics of animal population, Aust J. Zool., 2 (1954), 9-65.

[7] B.P. Smith, The potential of mites as biological control agents of mosquitoes, in "Research needs for development of biological control of pest by mites", (M. Hoy, G. Cunnigham and L. Knutson, eds.) pp. 79-85, Agric. Exp. Stn. Univ. Calif., USA, 1983.

[8] B.P. Smith, S.B. McIver, Factors influencing host selection and successful parasitism of Aedes spp. mosquitoes by Arrenurus spp. mites, Can J. Zool., 62 (1984), 1114-1120.

[9] I.M. Smith, D.R. Cook, Water mites, in "Freshwater invertebrates from North American" ( Thorp and Covich, eds.), pp. 470-492, Academic Press, USA, 1991.

[10] D.H. Stechmann, Zum Wirtskreis synopischer Arrenurus-Arten (Hydrachnellae, Acari) mit parasitischer Entwicklung an Nematocera (Diptera), Z. Parasitekd, 62 (1980), 267-283.

[11] F. Verhulst, "Nonlinear Differential Equations and Dynamical Systems", Springer-Verlag, New York, 1990. 